\title{
Lyotropic Liquid-Crystalline Mesophase of Lithium Triflate-Nonionic Surfactant as Gel Electrolyte for Graphene Optical Modulator
}

\author{
Fadime M. Balci, ${ }^{\dagger}$ Sinan Balci, ${ }^{*}{ }^{\star}$ Coskun Kocabas, ${ }^{*},{ }^{\circledR}$ and Ömer Dag, ${ }^{* \dagger}$ \\ ${ }^{\dagger}$ Department of Chemistry and UNAM, Bilkent University, 06800 Ankara, Turkey \\ ${ }^{\ddagger}$ Department of Astronautical Engineering, University of Turkish Aeronautical Association, 06790 Ankara, Turkey \\ ${ }^{\S}$ Department of Physics and UNAM, Bilkent University, 06800 Ankara, Turkey
}

\section{Supporting Information}

ABSTRACT: Lithium salt (noncoordinating anions, such as lithium triflate (Ltf)) gel electrolytes may be key for the practical use of electrochemical devices. We introduce a new lyotropic liquid-crystalline (LLC) mesophase using Ltf, a small amount of water (as low as 1.3 water per Ltf), and nonionic surfactant $\left(\mathrm{C}_{18} \mathrm{H}_{37}\left(\mathrm{OCH}_{2} \mathrm{CH}_{2}\right)_{10} \mathrm{OH}, \mathrm{C}_{18} \mathrm{E}_{10}\right)$. The LLC phase forms over a broad range of $\mathrm{Ltf} / \mathrm{C}_{18} \mathrm{E}_{10}$ mole ratios, 2-18. The clear ethanol solution of the ingredients can be either directly spin-coated over a glass substrate to form a gel phase or it can be prepared as a gel by mixing Ltf, water, and $\mathrm{C}_{18} \mathrm{E}_{10}$. The mesophase leaches out surfactant molecules at low salt concentrations, but a salt/surfactant mole ratio of above 8 , the phase is homogeneous with a cubic mesostructure, fully transparent in the visible optical region, mechanically flexible, and an effective gel electrolyte. We have observed a large electrostatic doping on graphene with the Fermi energy level of $\sim 1.0 \mathrm{eV}$ using $\mathrm{Ltf}-\mathrm{C}_{18} \mathrm{E}_{10}$ gel electrolytes. The Ltf-based gels demonstrate better properties than commonly used ionic liquid electrolyte in graphene optical modulators. The stability of the new gel electrolytes and their superior performance make them suitable electrolytes for use in graphene-based optical modulators.

\section{INTRODUCTION}

Lyotropic liquid-crystalline (LLC) mesophases consist of at least two components, such as a solvent and a surfactant. The solvent may be water, ${ }^{1,2}$ water-oil, ${ }^{3}$ aqueous solutions, ${ }^{4}$ ionic liquids, ${ }^{5-7}$ acids, ${ }^{8,9}$ and salts. ${ }^{10-12}$ The salts can be alkali metals $\left(\mathrm{LiX}, \mathrm{X}\right.$ is $\left.\mathrm{NO}_{3}{ }^{-}, \mathrm{Cl}^{-}, \mathrm{Br}^{-}, \mathrm{I}^{-}\right),{ }^{11,12}$ alkaline earth metals $\left(\mathrm{CaX}_{2}\right.$, $\mathrm{X}^{-}$is $\mathrm{Cl}^{-}$and $\left.\mathrm{NO}_{3}{ }^{-}\right),{ }^{12}$ transition metals $\left(\left[\mathrm{M}\left(\mathrm{H}_{2} \mathrm{O}\right)_{n}\right]\left(\mathrm{NO}_{3}\right)_{2}\right.$, where $\mathrm{M}$ represents $\mathrm{Mn}(\mathrm{II}), \mathrm{Co}(\mathrm{II}), \mathrm{Ni}(\mathrm{II}), \mathrm{Zn}(\mathrm{II})$, and $\mathrm{Cd}(\mathrm{II})),{ }^{10}$ or lanthanides (Eu(II) and La(III)). ${ }^{13}$ The common properties of these salts are their low melting point or high solubility. ${ }^{11,12}$ It is also important to note that the melting points of the salts drop ${ }^{10}$ and their solubility is enhanced ${ }^{11,12}$ in confined spaces, such as in the hydrophilic domains of the mesophases, known as soft confinement effect. ${ }^{14}$ Even though most lithium salts have a high melting point, because of their high solubility, they form LLC phases with nonionic surfactants. ${ }^{11,12}$

The salt-surfactant LLC mesophases are important in the development of gel-electrolytes for practical electrochemical devices, ${ }^{15}$ such as solar cells, ${ }^{16}$ batteries, ${ }^{17}$ supercapacitors, ${ }^{8}$ and so on. Therefore, it is important to investigate the saltsurfactant mesophases, especially the lithium salt-surfactant mesophases, toward the development of gel-electrolytes for electrochemical systems. Moreover, the lithium salts of noncoordinating anions (such as $\mathrm{BF}_{4}^{-}, \mathrm{PF}_{6}^{-}$, and $\mathrm{CF}_{3} \mathrm{SO}_{3}^{-}$) are very important due to their high ionic conductivity. ${ }^{18-20}$ However, the supersaturated solutions of lithium salts of these ions have not been investigated as a solvent in the saltsurfactant LLC mesophases. For instance, $\mathrm{LiCF}_{3} \mathrm{SO}_{3}$ is a very soluble salt in water and can be a good candidate for a stable LLC mesophase, which may be used as the gel-electrolyte in various super capacitors and battery systems.

For example, a phosphoric acid-surfactant mesophase, used as a gel-electrolyte, has been recently tested in a graphene optical modulator, a supercapacitor consisting of two graphene electrodes and a gel-electrolyte, with excellent results. Polarizing the electrolyte by an applied electric field between the two graphene electrodes effectively dopes the graphene and significantly shifts its Fermi level to effectively modulate the optical transmittance of graphene. ${ }^{8,21}$ Major challenges in the device configuration include finding an electrolyte that (i) has a large electrochemical window, (ii) shifts the Fermi level of graphene greater than $1 \mathrm{eV}$ for visible optoelectronic applications, (iii) reaches very large charge densities on graphene, (iv) is transparent in the visible region, and (v) is mechanically flexible for solid state device applications. For these reasons, ionic liquids have been considered target materials. ${ }^{21}$ However, gel-electrolytes that perform as well as ionic liquids may be more beneficial for use in practical applications.

Received: April 18, 2017

Revised: May 11, 2017

Published: May 12, 2017 
In this contribution, we have developed a new LLC mesophase of $\mathrm{LiCF}_{3} \mathrm{SO}_{3}-\mathrm{C}_{18} \mathrm{H}_{37} \mathrm{OCH}_{2} \mathrm{CH}_{2) 10} \mathrm{OH}$ (denoted as $\mathrm{Ltf}-\mathrm{C}_{18} \mathrm{E}_{10}$ ) that can be used as a gel electrolyte in graphene optical modulators. The mesophase is very stable over a broad range of $\mathrm{Ltf} / \mathrm{C}_{18} \mathrm{E}_{10}$ mole ratios, characterized using various techniques (such as XRD, POM, FTIR, and conductivity measurements). Its performance, as LLC gel electrolytes, has been demonstrated in an optical modulation of graphene in the visible region. The new electrolyte developed here, applicable in graphene optical modulators, has many advantages such as ease of device fabrication, minimal electrolyte leakage, excellent electrochemical properties, high charge density on graphene, mechanical flexibility, produced under ambient conditions, transparent in the visible region, stable over long periods (more than 3 months as measured in this study), and so on.

\section{EXPERIMENTAL PART}

Preparation of $\mathrm{Ltf}-\mathrm{C}_{18} \mathrm{E}_{10}$ Solutions. In a typical preparation, the required amount of Ltf was first dissolved in ethanol, and following that the required amount of $\mathrm{C}_{18} \mathrm{E}_{10}$ was added and stirred overnight at RT to obtain a clear solution. For example, the sample with $2 \mathrm{Ltf} / \mathrm{C}_{18} \mathrm{E}_{10}$ was prepared as follows. First, $2 \mathrm{mmol}$ (312 mg) of Ltf was dissolved in $5 \mathrm{~g}$ EtOH. Subsequently, $1 \mathrm{mmol}(711 \mathrm{mg})$ of $\mathrm{C}_{18} \mathrm{E}_{10}$ was added to the above solution and stirred overnight to obtain a homogenized solution. The clear solutions were then coated on glass slides via spin coating at $1000 \mathrm{rpm}$ for $15 \mathrm{~s}$ to obtain LLC mesophases.

Preparation of Ltf- $\mathrm{C}_{18} \mathrm{E}_{10}$ LLC Mesophases. The required amounts of Ltf, water, and $\mathrm{C}_{18} \mathrm{E}_{10}$ were placed in a vial and sealed using Teflon tape. As an example, the sample with $4 \mathrm{Ltf} / \mathrm{C}_{18} \mathrm{E}_{10}$ and $3 \mathrm{H}_{2} \mathrm{O} / \mathrm{Ltf}$ was prepared by mixing 4 mmol $(624 \mathrm{mg})$ of Ltf, $1 \mathrm{mmol}(711 \mathrm{mg})$ of $\mathrm{C}_{18} \mathrm{E}_{10}$, and 12 $\mathrm{mmol}(216 \mathrm{mg})$ of $\mathrm{H}_{2} \mathrm{O}$ in a vial. The vial was kept at $80^{\circ} \mathrm{C}$ in a water bath for $24 \mathrm{~h}$ to obtain homogenized gels.

Synthesis of Graphene. Graphene layers on ultrasmooth copper foil substrates (Mitsui Mining and Smelting Company, B1-SBS) were synthesized using chemical vapor deposition (CVD), as described in our previous publications. ${ }^{8,21}$ We are able to synthesize single and multilayer graphene in a large area (several $\mathrm{cm}^{2}$ ). During the annealing step, the copper oxide on the copper substrate was removed by sending $\mathrm{H}_{2}$ gas with a flow rate of $100 \mathrm{sccm}$. The graphene layer on the copper substrate was synthesized at $1035^{\circ} \mathrm{C}$ and 5 Torr under a $\mathrm{CH}_{4}$ flow rate of $10 \mathrm{sccm}$. After $30 \mathrm{~s}$ of growth, the growth was stopped and cooled to room temperature.

AC Conductivity Measurements. AC conductivity measurements were taken using a Gamry G750 potentiostat/ galvanostat operating at an AC voltage of $10 \mathrm{mV}$ and $100 \mathrm{kHz}$. The FTO glass electrodes were prepared as described in our previous publications. ${ }^{16}$ The cell constant was determined using standard $\mathrm{KCl}$ solutions with a known conductivity, each time before the measurement of the gels. The solutions were dropped over the prestretched line over FTO glass. Upon evaporation of the solvent $(\sim 24 \mathrm{~h})$, the counter and reference electrodes were attached to one side and working electrode was attached to the other side for the conductivity measurements.

XRD Measurements. Small-angle XRD patterns were collected using Miniflex diffractometer, equipped with highpower $\mathrm{Cu} \mathrm{K} \alpha$ source operating at $30 \mathrm{kV} / 15 \mathrm{~mA}$ and wavelength of $1.5405 \AA$. Film samples that were spin-coated over glass slides were used for the XRD measurements.
ATR-FTIR Measurements. The FTIR spectra were recorded using a Bruker Alpha Platinum ATR in the 400$4000 \mathrm{~cm}^{-1}$ with a $4 \mathrm{~cm}^{-1}$ resolution and conducting 32 scans. A few drops of solution were placed on the diamond ATR crystal, and the spectra were followed for 2 days until all ethanol was evaporated and no spectral changes were identified.

Determination of Water Amounts in the Gels. Around $1 \mathrm{~g}$ of the clear solution was placed onto a plastic tray and the mass of the solution was monitored for 2 days until there was no significant change in the mass of the sample.

Optical and Electrical Measurements. Graphene layers were transferred to glass substrates; then, graphene-based optical supercapacitors ${ }^{21}$ were fabricated. The graphene layers synthesized on a copper substrate were covered with Shipley 1813 photoresist liquid and then annealed at $60^{\circ} \mathrm{C}$ overnight. To transfer the graphene from photoresist layer to a glass surface, the copper film attached to the graphene was chemically etched in $1 \mathrm{M} \mathrm{FeCl}_{3}$ aqueous solution and then washed with excessive amount of water. Subsequently, the graphene layers attached to the photoresist were placed on a glass surface and heated to $80{ }^{\circ} \mathrm{C}$ for a few minutes on a hot plate. Following this the photoresist layer was removed with acetone and washed with an ample amount of isopropanol. Another glass substrate with a $\sim 100 \mathrm{~nm}$ gold film and graphene-covered glass substrate were then joined. The counter electrodes were separated by a $100 \mu \mathrm{m}$ thick plastic spacer, a double-sided adhesive tape stripe. The space between the graphene and gold electrodes was filled with an electrolyte. The ionic liquid electrolyte used in this study is diethylmethyl(2methoxyethyl) ammonium bis(trifluoromethylsulfonyl)imide. Under the applied bias voltage, the electrolyte generates electrical double layer (EDL) at the interface between graphene and electrolyte. The optical properties (transmission) of singlelayer graphene were measured in the visible and near-infrared wavelength as a function of the applied bias voltage using a Fourier transform infrared spectrometer (Bruker VERTEX 70v). Lithium-salt-based LLC gel electrolytes were inserted into the graphene supercapacitor by heating the device and electrolyte to $70{ }^{\circ} \mathrm{C}$. $\sim 20 \mu \mathrm{L}$ of the synthesized electrolyte was placed at the edge of the device; then, the electrolyte flows in the narrow space between the electrodes due to the capillary forces; see Figure S1 for the details. Capacitance and total resistance of the graphene capacitor were measured by using LCR Meter (Hewlett-Packard, 4284A). Bias voltages to graphene and gold electrodes were applied by using a Source Meter (Keithley, 2400).

\section{RESULTS AND DISCUSSION}

The LLC mesophases of lithium triflate $\left(\mathrm{LiCF}_{3} \mathrm{SO}_{3}, \mathrm{Ltf}\right)$ and brij $76\left(\mathrm{C}_{18} \mathrm{H}_{37}\left(\mathrm{OCH}_{2} \mathrm{CH}_{2}\right)_{10} \mathrm{OH}, \mathrm{C}_{18} \mathrm{E}_{10}\right)$ form over a broad range of salt/surfactant mole ratios $(2-18$, denoted as \#-Ltf$\mathrm{C}_{18} \mathrm{E}_{10}$, where \# represents Ltf $/ \mathrm{C}_{18} \mathrm{E}_{10}$ mole ratio), corresponding to a ratio as high as $82 \%(\mathrm{w} / \mathrm{w})$. This indicates that a major component of the mesophase could be the salt species. The mesophases can be prepared as a solution in excess ethanol, which can be completely evaporated upon spin coating over any substrate to obtain the gel LLC phase or prepared as a gel starting from salt, surfactant, and small amount of water. In general, when using the second method, it is more difficult to produce a homogeneous mixture of the LLC phase; however, it is more practical to use as a gel electrolyte in electrochemical applications. 
a)

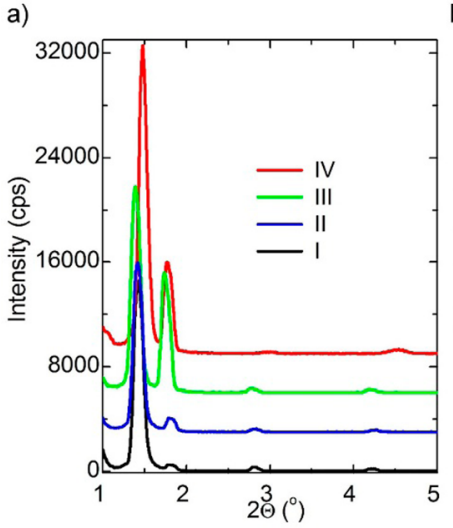

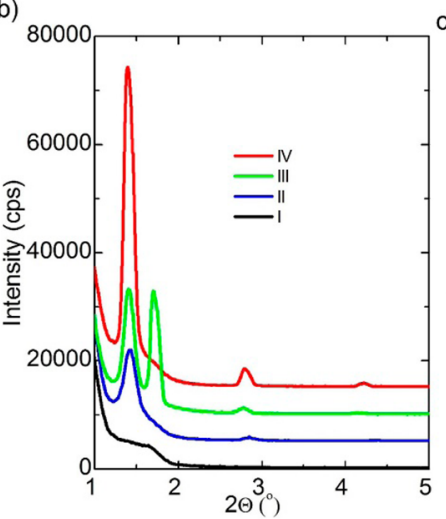

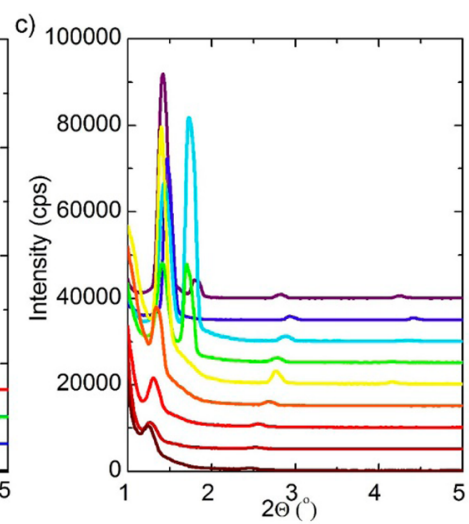

Figure 1. XRD pattern of (a) 2-Ltf- $\mathrm{C}_{18} \mathrm{E}_{10}$ with aging (I) fresh, (II) 1 day, (III) 1 week, and (IV) 2 weeks. (b) 8-Ltf- $\mathrm{C}_{18} \mathrm{E}_{10}$ mole ratio with aging (I) fresh, (II) 1 day, (III) 1 week, and (IV) 5 weeks. (c) \#-Ltf-C ${ }_{18} \mathrm{E}_{10}$ with increasing \# from 2 (top) to 18 (bottom) with an increment of 2.
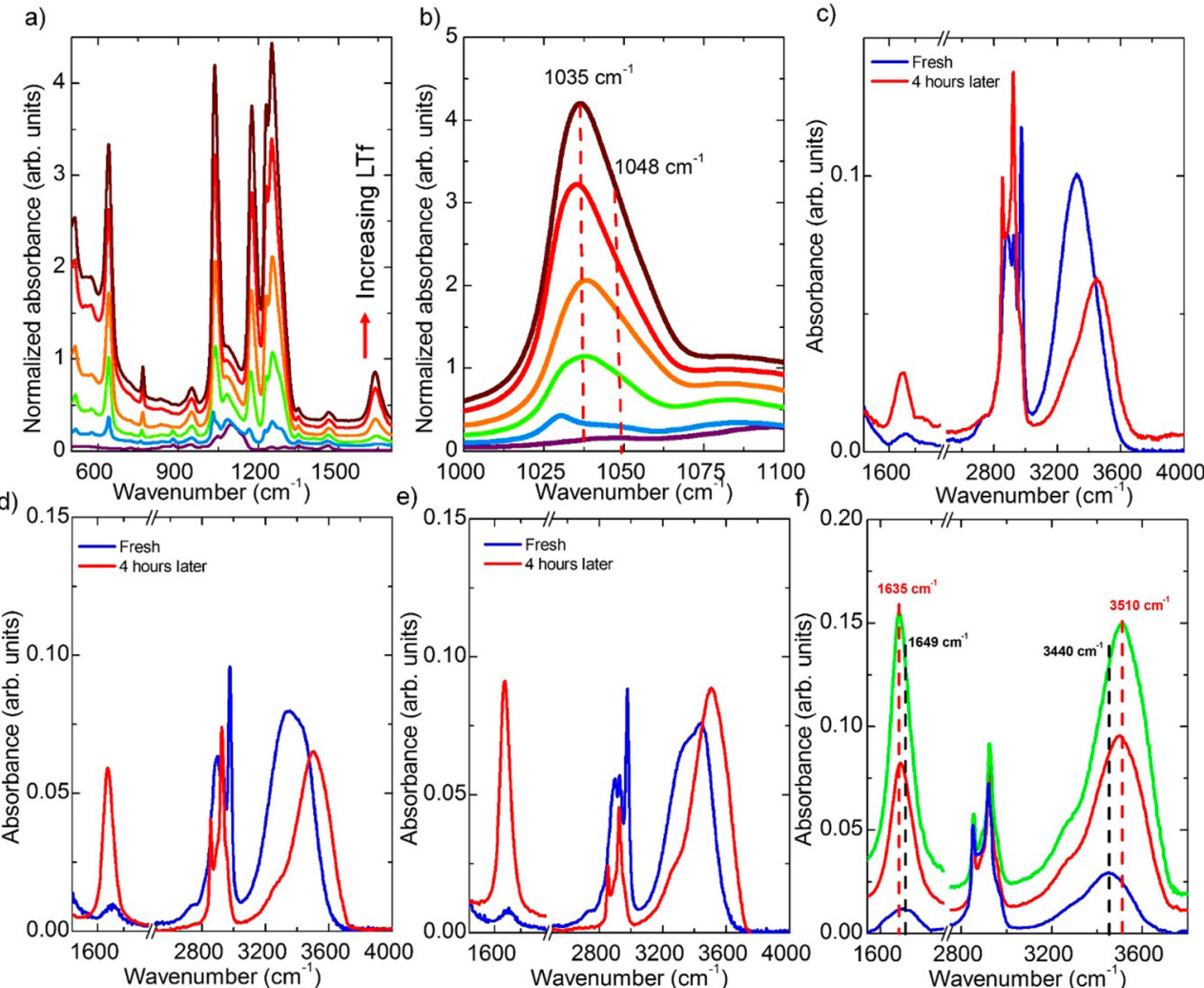

Figure 2. Normalized ATR-FTIR spectra of $(\mathrm{a}, \mathrm{b})$ \#-Ltf- $\mathrm{C}_{18} \mathrm{E}_{10}$ with increasing Ltf (Ltf/C $\mathrm{C}_{18} \mathrm{E}_{10}$ mole ratio increases from 2 to 18 with four increments, bottom is the spectrum of pure $\mathrm{C}_{18} \mathrm{E}_{10}$ ). ATR-FTIR spectra of $x \mathrm{Ltf} / \mathrm{C}_{18} \mathrm{E}_{10}$ during aging: $x$ is (c) 2, (d) 10, and (e) 18 (fresh and $4 \mathrm{~h}$ ). (f) Normalized spectra after $24 \mathrm{~h}$ of aging of the same samples.

Both mesophases, prepared as gels and solutions, were characterized using POM, XRD, and FTIR techniques to elucidate the structural details as well as AC conductivity measurements for the electrical characterization. Figure 1 shows a series of small-angle XRD patterns of samples with different concentration of Ltf. Notice that both films, prepared by spin coating or spreading over a substrate, diffract at small angles with no diffraction at high angles. In fact, both preparation methods provide the same LLC phase upon aging.

The samples, prepared using ethanol solution, have been monitored by recording their XRD patterns over 5 weeks to ensure homogeneous LLC phase through complete ethanol evaporation. Figure $\mathrm{a} a, \mathrm{~b}$ shows the aging process of two samples, namely, 2 and $8 \mathrm{Ltf}$ and $1 \mathrm{C}_{18} \mathrm{E}_{10}$. The diffraction lines observed immediately after spin coating undergo some changes, while keeping the line position relatively constant. At low Ltf concentrations, there are two sets of diffraction lines at small angles, which eventually become a single set at higher concentrations; see Figure 1. The small-angle XRD pattern of 2 -Lt- $\mathrm{C}_{18} \mathrm{E}_{10}$ displays lines at $1.48,1.77,2.99$, and $4.54^{\circ}, 2 \theta$ values. The diffraction line at $1.77^{\circ}$ likely corresponds to a free surfactant, which gradually disappears at higher Ltf concentrations. The free surfactant line appears upon coating a clear solution of the salt-surfactant, gradually increases after $1 \mathrm{~h}$, and then gradually decreases over time with further aging the gel films. It is likely that the mesophase leaches out crystalline 
surfactants, which gradually become disordered or molten over time due to the thermal energy (melting point of free surfactant is $\sim 38{ }^{\circ} \mathrm{C}$ ). Note also that the XRD pattern of the free surfactant, spin-coated from its clear ethanol solution, diffracts at $\sim 1.77^{\circ}$, which also gradually disappears over time, similar to our samples. Besides, the surfactant leach from the LLC media has also been observed in other lithium salt-pluronic LLC phases. $^{22}$

The 2-Ltf- $\mathrm{C}_{18} \mathrm{E}_{10}$ sample has also been monitored using POM, and it displays birefringent and dark domains, likely due to the free surfactant and LLC domains, respectively. It is worth noting that the freshly prepared films, before surfactant separation, display focal conic fan texture under POM. It means that the mesophase is $2 \mathrm{D}$ hexagonal at low salt concentrations but is metastable and undergoes phase separation into salt-rich LLC and salt-free surfactant domains over time. Therefore, the salt concentration in the LLC domains is much higher than $2 \mathrm{Ltf} / \mathrm{C}_{18} \mathrm{E}_{10}$. The free surfactant line gradually disappears with an increasing Ltf/ $\mathrm{C}_{18} \mathrm{E}_{10}$ mole ratio in the samples, and at a mole ratio above $8 \mathrm{Ltf} / \mathrm{C}_{18} \mathrm{E}_{10}$, all diffraction lines can be indexed to a cubic mesophase. Also, this behavior correlates with the POM images that appear dark between the two crossed polarizers. Note that the diffraction lines gradually shift to lower angles with an increasing Ltf/ $\mathrm{C}_{18} \mathrm{E}_{10}$ mole ratio. If we consider the line at $\sim 1.34^{\circ}, 2 \theta$, originating from the (200) plane of the cubic phase, the unit -cell parameter increases from 120 to $142 \AA$ by increasing Ltf/ $\mathrm{C}_{18} \mathrm{E}_{10}$ mole ratio from 2 to 18 , respectively; see Figure 1c. However, the changes of the unit-cell parameters, at low Ltf/ $\mathrm{C}_{18} \mathrm{E}_{10}$ ratio, are negligibly small and gradual at higher ratios. It is likely that the $\mathrm{Ltf} / \mathrm{C}_{18} \mathrm{E}_{10}$ ratio in the LLC mesophase is constant to a mole ratio around 8 and then gradually increases at higher salt concentrations, where the extra surfactant molecules in the low Ltf concentrations are leached out from the media to compensate for a constant salt/surfactant ratio.

Furthermore, the salt-surfactant mixtures display mesophases in the following order $\mathrm{V}_{1}$ (bicontinuous cubic), $\mathrm{H}_{1}$ (normal hexagonal), and $\mathrm{I}_{1}$ (micellar cubic), with a dominating hexagonal phase, with an increasing salt/surfactant mole ratio. Therefore, it is reasonable to conclude that the hexagonal phase of the $\mathrm{Ltf}-\mathrm{C}_{18} \mathrm{E}_{10}$ system is metastable and undergoes a phase separation into micellar cubic phase and free surfactant at low Ltf $/ \mathrm{C}_{18} \mathrm{E}_{10}$ mole ratios. Besides, the diffraction patterns in Figure 1c show some variations during the aging process; however, all diffraction lines are indexed to a micellar cubic phase, where the lines at $1.39,1.70,2.77$, and $4.16^{\circ}, 2 \theta$, correspond to (200), (211), (400), and (600) planes, respectively. The line, corresponding to the (200) plane, remains in all of the samples but gradually loses its intensity and shifts to smaller angles due to increase in unit-cell size in the mesophase to accommodate the excess salt species. Interestingly, the mesophase in the $\mathrm{Lft}-\mathrm{C}_{18} \mathrm{E}_{10}$ is cubic in all compositions, which is quite unusual for salt surfactant mesophases.

In addition, we recorded the ATR-FTIR spectra of a series of samples during solvent evaporation with different $\mathrm{Ltf} / \mathrm{C}_{18} \mathrm{E}_{10}$ mole ratios. Figure $2 \mathrm{a}, \mathrm{b}$ shows a series of spectra after aging the samples for $24 \mathrm{~h}$, where the mesophase no longer undergoes changes. The peaks due to the Ltf species increase with an increasing salt concentration in the media. However, it is difficult to spectroscopically quantify the free surfactant in the media because the spectrum of the surfactant changes to the final spectrum upon the addition of as little as 0.1 salt/ surfactant to the media. This means that the initial conformation of the ethylene oxide units changes in the presence of a 0.1 salt per surfactant, and the rest is an assembly of the surfactant with the help of $\mathrm{LiCF}_{3} \mathrm{SO}_{3} x \mathrm{H}_{2} \mathrm{O}$. Notably, the Ltf peaks dominate the spectra. For instance, the sharp peak at $1035 \mathrm{~cm}^{-1}$ is known to originate from the $\nu_{\mathrm{s}}\left(\mathrm{SO}_{3}\right)$ mode of the free triflate ion, and the tail on the high-energy site of this peak corresponds to the same mode in an ion pair. Figure $2 \mathrm{~b}$ clearly shows that the triflate species are predominantly free ions. This is important for the ionic conductivity of the gel phase; see the latter. The intense peaks at $640,1176,1232$, and $1255 \mathrm{~cm}^{-1}$, due to $\delta\left(\mathrm{SO}_{3}\right), \nu_{\text {as }}\left(\mathrm{CF}_{3}\right), \nu_{\mathrm{s}}\left(\mathrm{CF}_{3}\right)$, and $\nu_{\text {as }}\left(\mathrm{SO}_{3}\right)$, respectively, and a tail on the high-energy side of $1255 \mathrm{~cm}^{-1}$, broken into two components at $\sim 1272$ and $\sim 1300 \mathrm{~cm}^{-1}$, originate from the asymmetric stretching mode of the $\mathrm{SO}_{3}$ unit of the free and coordinated triflate ion, respectively. ${ }^{23,24}$

Figure $2 \mathrm{c}-\mathrm{f}$ shows the surfactant $\nu \mathrm{CH}$ stretching and waterbending and -stretching regions of three samples in two different stages of the aging process. The fresh samples display weak water bending at $\sim 1649 \mathrm{~cm}^{-1}$ and a broad waterstretching feature at $3440 \mathrm{~cm}^{-1}$ with a shoulder around 3200 $\mathrm{cm}^{-1}$, characteristic of the bulk water (hydrogen-bonded network of water). The $\nu \mathrm{CH}$ stretching region displays peaks related to the surfactant molecules and ethanol. However, upon complete evaporation of ethanol, both the bending and stretching modes of the water display drastic changes; compare the spectra in Figure $2 c-e$. The bending mode gradually red shifts and becomes more intense and sharper, while stretching modes are blue-shifting, from 1649 to $1635 \mathrm{~cm}^{-1}$ and 3440 to $3510 \mathrm{~cm}^{-1}$, respectively, indicating that the water is becoming free or hydration-only water; ${ }^{25}$ compare the spectra in Figure $2 \mathrm{f}$. The bending mode also becomes sharper with an increasing Ltf in the media. The stretching to bending intensity ratio also displays drastic changes, from 2.5 to 1.0 increasing from 2 to $18 \mathrm{Ltf} / \mathrm{C}_{18} \mathrm{E}_{10}$ mole ratio, likely indicating the change in the bulk/hydration water ratio in the media. To support this observation and also to determine the water content of the samples, we monitored the weight change over a four-digit balance during ethanol evaporation of the solutions. Owing to the very fast evaporation of ethanol, the mass of the solution decays exponentially; see Figure 3a. After mesophase formation, the mass of the solution becomes constant. Indeed, the LLC mesophase stabilizes at around $2 \mathrm{H}_{2} \mathrm{O} / \mathrm{Ltf}$ for both 2-Ltf- $\mathrm{C}_{18} \mathrm{E}_{10}$

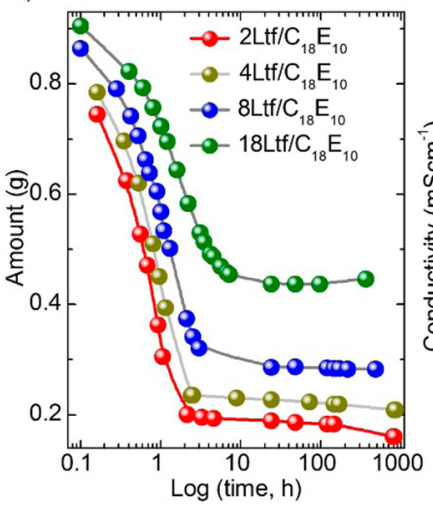

b)

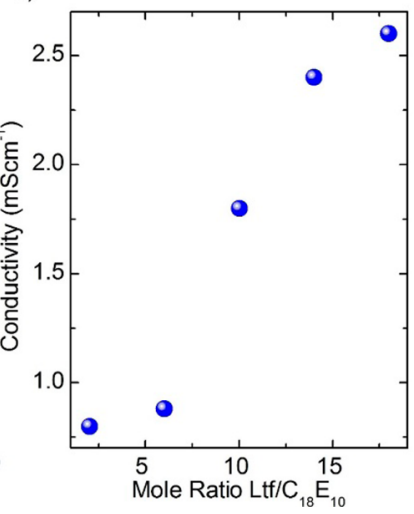

Figure 3. Variation of (a) the mass and (b) the conductivity of the samples with a Ltf $/ \mathrm{C}_{18} \mathrm{E}_{10}$ mole ratio of between 2 and 18 . 
a)

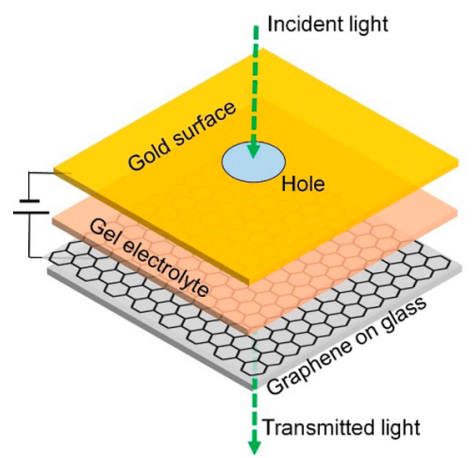

b)

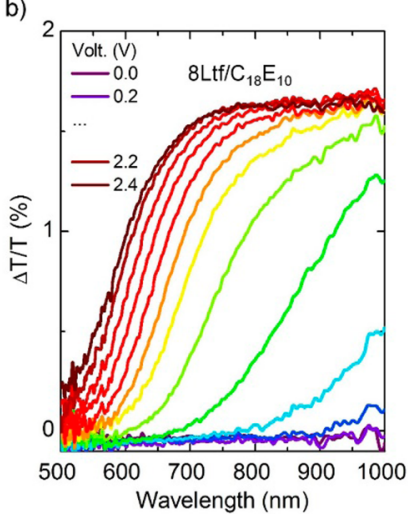

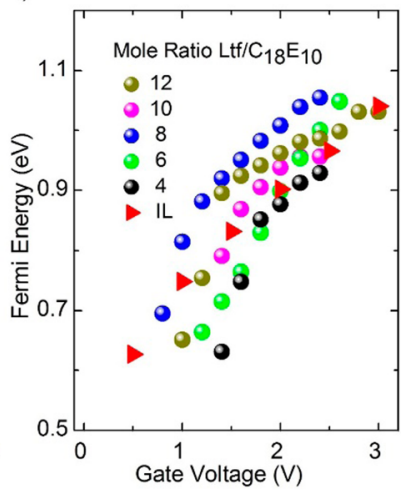

Figure 4. Application of Ltf based LLCs gel electrolytes for graphene optical modulators. (a) Schematic representation of a graphene-gold supercapacitor filled with Ltf salt-based liquid-crystalline gel electrolyte. The LLC system has Ltf/ $\mathrm{C}_{18} \mathrm{E}_{10}$ mole ratio of 8. (b) Variation of normalized transmission of the graphene supercapacitor filled with Ltf salt-based liquid-crystalline gel electrolyte versus the wavelength of the incident light for various applied bias voltages. (c) Variation of Fermi energy level of graphene as a function of applied bias voltages for varying $\mathrm{Ltf} / \mathrm{C}_{18} \mathrm{E}_{10} \mathrm{~mole}$ ratio in LLC gel electrolytes and typical ionic liquid used in graphene optical modulators.

and 4 -Ltf- $\mathrm{C}_{18} \mathrm{E}_{10}$ and at around $1.3 \mathrm{H}_{2} \mathrm{O} / \mathrm{Ltf}$ for both 8 -Ltf$\mathrm{C}_{18} \mathrm{E}_{10}$ and 18-Ltf- $\mathrm{C}_{18} \mathrm{E}_{10}$.

Remarkably, the water/Ltf mole ratio decreases to almost 1.3 around $8 \mathrm{Lft} / \mathrm{C}_{18} \mathrm{E}_{10}$ and remains constant above a mole ratio of 8 . These observations are consistent with the behaviors observed in the XRD patterns as well as the discussions in the XRD Measurements section. Therefore, it is reasonable to conclude that the Ltf- $\mathrm{C}_{18} \mathrm{E}_{10}$ system is metastable up to an 8 Ltf $/ \mathrm{C}_{18} \mathrm{E}_{10}$ mole ratio and leaches out surfactant with some bulk-like water.

Additionally, we recorded the AC conductivity of a set of \#-Lft- $\mathrm{C}_{18} \mathrm{E}_{10}$ during aging using an FTO substrate that was designed for this purpose. ${ }^{16}$ The AC ionic conductivity increases slowly in samples with a low Lft concentration and increases gradually after a mole ratio of 6 ; see Figure $3 \mathrm{~b}$. Notably, the Ltf- $\mathrm{C}_{18} \mathrm{E}_{10}$ mesophase displays the highest $\mathrm{AC}$ ionic conductivity among all lithium salt-surfactant systems investigated in the literature. ${ }^{12,22}$ The highest conductivity was reported for the $18-\mathrm{Lft}-\mathrm{C}_{18} \mathrm{E}_{10}$ to be $2.6 \mathrm{mS} / \mathrm{cm}$. Notice that there is a factor of 3 compared with the polyelectrolytes of $\mathrm{Lft}$ using poly(ethylene oxide)s. ${ }^{26-28}$ There, the Lft has a limited solubility and also a stronger ion-ethylene oxide interaction that likely reduces the mobility of lithium ion in the media. However, in the lithium salt-surfactant systems, the salt is in the molten phase in the presence of a small amount of water with better ionic conductivity. It is likely that the lithium ion mobility is also enhanced in the confined space due to thermodynamic effects that need to be further investigated. ${ }^{29,30}$

We employed the Ltf- $\mathrm{C}_{18} \mathrm{E}_{10}$ LLC mesophase as a gel electrolyte in a graphene optical modulator to evaluate the electro-optical performance of the LLC gels. For this purpose, five compositions were chosen with an Ltf $/ \mathrm{C}_{18} \mathrm{E}_{10}$ mole ratio of $4,6,8,10$, and 12. Figure 4a displays the schematic representation of the graphene-based supercapacitor structure used as an optical modulator. A gold film on a glass surface and graphene layer on an another glass surface form the capacitor structure (Figure S1). The electrodes are separated by an insulating spacer layer. The gel electrolyte was placed between the capacitor plates by heating to a temperature higher than gel electrolyte's melting point $\left(\sim 75^{\circ} \mathrm{C}\right)$ and then placing the liquid electrolyte on preheated graphene capacitor device; see Figure S1. It should be noted that the graphene-gold supercapacitor works by ion gating. Owing to the electric double-layer (EDL) formation, the electric field is screened very close to the graphene surface (only a few nanometers) by the mobile ions in the medium. The fractional variation of the transmission of the incident light through the device as a function of the applied gate voltages is shown in Figure $4 \mathrm{~b}$ and Figure S2. It should be noted that the ideal suspended graphene in air absorbs only $\Pi \alpha$ $=2.3 \%$ ( $\alpha$ is the fine structure constant) of the incident light. ${ }^{31}$ When graphene is placed on a substrate such as a glass substrate in our case, the graphene absorbs $1.45 \%$ (corrected absorption $=\Pi \alpha(2 /(1+n)),{ }^{2}$ where $n$ is the refractive index of BK7 glass at $600 \mathrm{~nm}, 1.5163)$ of the incident light, ${ }^{32}$ which is in close agreement with our observed variation $(\sim 1.6 \%)$ in Figure 4b. This value should decrease with increasing the defect density on graphene. Because of the Pauli blocking, the graphene becomes transparent for incoming light at energies smaller than twice the Fermi level shift. ${ }^{33}$ Note that the Pauli blocking happens when the final optical transition states are occupied because of the increase in the charge density on graphene. The observed Fermi level shift increases linearly with the applied gate voltages. The mole ratio of the $\mathrm{Ltf} / \mathrm{C}_{18} \mathrm{E}_{10}$ gel electrolyte used in Figure $4 \mathrm{~b}$ is 8 . Also, a series of samples with varying composition of $\mathrm{Ltf} / \mathrm{C}_{18} \mathrm{E}_{10}$ mole ratio were prepared and their electro-optical performances have been tested (Figure $4 c)$. Remarkably, we observed that the Ltf/ $\mathrm{C}_{18} \mathrm{E}_{10}$ mole ratio of 8 has the best performance in the graphene optical modulators. In addition, the fabricated device showed a similar performance after 3 months; see Figure S6. The capacitance and resistance of all compositions have been measured (see the Supporting Information, Figures S2-S6). In addition to the Ltf salt, we also prepared and used $\mathrm{LiCl}, \mathrm{LiBr}$, and $\mathrm{LiNO}_{3}$ salt-based LLC mesophases as electrolytes in graphene supercapacitors. However, we were only able to insert an $\mathrm{LiCl}$-based electrolyte (due to high melting points of those mesophases) into the device, which performed very weakly when compared with the Ltf-based gel electrolytes; see Figure S2f. Figure 4c demonstrates the optical performance of all $\mathrm{Ltf} / \mathrm{C}_{18} \mathrm{E}_{10}$ compositions and a commonly used ionic liquid in graphene supercapacitors; see Figure S7 as well. We observed that an Ltf/ $\mathrm{C}_{18} \mathrm{E}_{10}$ mole ratio of 8 displays the best performance in graphene optical modulators. 


\section{CONCLUSIONS}

A new LLC mesophase of $\operatorname{Ltf}-\mathrm{C}_{18} \mathrm{E}_{10}$ has been prepared, characterized, and employed as a gel electrolyte in graphenebased optical modulators. A new LLC system consists of Ltf with a very small amount of water (as low as 1.3 water molecules per Ltf) and a nonionic surfactant $\left(\mathrm{C}_{18} \mathrm{E}_{10}\right)$. The LLC mesophase forms over a broad range of $\mathrm{Ltf} / \mathrm{C}_{18} \mathrm{E}_{10}$ mole ratios $(2-18)$. The first step in the preparation is to obtain a clear ethanol solution of Ltf and $\mathrm{C}_{18} \mathrm{E}_{10}$. After obtaining the solution, the solvent, ethanol, is evaporated by spin coating over a glass substrate, and the gel phase is immediately formed. The mesophase is transparent in the visible region of the electromagnetic spectrum, mechanically flexible, and stable over a broad range of $\mathrm{Ltf} / \mathrm{C}_{18} \mathrm{E}_{10}$ mole ratios. The LLC gel electrolyte displays good ionic conductivity and an excellent electro-optical performance in the graphene-based optical modulators. Maximum Fermi energy shift in graphene is observed in the $8 \mathrm{Ltf} / \mathrm{C}_{18} \mathrm{E}_{10}$. The LLC gel electrolyte has many advantages in terms of device fabrication, electrolyte leakage, electrochemical properties, etc. The results pave the way for the development of gel electrolytes, which can increase the Fermi energy level shift of graphene to allow for the realization of graphene-based optoelectronics in the visible region of the electromagnetic spectrum.

\section{ASSOCIATED CONTENT}

\section{S Supporting Information}

The Supporting Information is available free of charge on the ACS Publications website at DOI: 10.1021/acs.jpcc.7b03622.

Schematic representation and photographs of the electrodes. More electrical and optical characterization data of the electrodes with gel electrolytes and ionic liquid. (PDF)

\section{AUTHOR INFORMATION}

\section{Corresponding Authors}

*S.B.: E-mail: sbalci@thk.edu.tr.

*C.K.: E-mail: ckocabas@fen.bilkent.edu.tr.

*O.D.: E-mail: dag@fen.bilkent.edu.tr.

ORCID $\odot$

Coskun Kocabas: 0000-0003-0831-5552

Ömer Dag: 0000-0002-1129-3246

Notes

The authors declare no competing financial interest.

\section{ACKNOWLEDGMENTS}

We thank the Scientific and Technological Research Council of Turkey (TÜBITAK) under project numbers $13 \mathrm{~F} 278$ and $215 Z 193$ for the financial support and Dr. Osman Balci (Bilkent University) for his fruitful discussions. O.D. is a member of the Science Academy, Istanbul, Turkey.

\section{REFERENCES}

(1) Mitchell, D. J.; Tiddy, G. J. T.; Waring, L.; Bostock, T.; Mcdonald, M. P. Phase-Behavior of Polyoxyethylene Surfactants with Water-Mesophase Structures and Partial Miscibility (Cloud Points). J. Chem. Soc., Faraday Trans. 1 1983, 79, 975-1000.

(2) Dong, R. H.; Hao, J. C. Complex Fluids of Poly(oxyethylene) Monoalkyl Ether Nonionic Surfactants. Chem. Rev. 2010, 110, 49785022.

(3) Alexandridis, P.; Olsson, U.; Lindman, B. A Record Nine Different Phases (Four Cubic, Two Hexagonal, and One Lamellar
Lyotropic Liquid Crystalline and Two Micellar Solutions) in a Ternary Isothermal System of an Amphiphilic Block Copolymer and Selective Solvents (Water and Oil). Langmuir 1998, 14, 2627-2638.

(4) Zheng, L. Q.; Minamikawa, H.; Harada, K.; Inoue, T.; Chernik, G. G. Effect of Inorganic Salts on the Phase Behavior of an Aqueous Mixture of Heptaethylene Glycol Dodecyl Ether. Langmuir 2003, 19, 10487-10494.

(5) Tang, J.; Li, D.; Sun, C. Y.; Zheng, L. Z.; Li, J. H. Temperature Dependant Self-assembly of Surfactant Brij 76 in Room Temperature Ionic Liquid. Colloids Surf., A 2006, 273, 24-28.

(6) Ichikawa, T.; Yoshio, M.; Hamasaki, A.; Mukai, T.; Ohno, H.; Kato, T. Self-organization of Room-temperature Ionic Liquids Exhibiting Liquid-crystalline Bicontinuous Cubic Phases: Formation of Nano-ion Channel Networks. J. Am. Chem. Soc. 2007, 129, 1066210663.

(7) Greaves, T. L.; Drummond, C. J. Ionic Liquids as Amphiphile Self-assembly Media. Chem. Soc. Rev. 2008, 37, 1709-1726.

(8) Tunkara, E.; Albayrak, C.; Polat, E. O.; Kocabas, C.; Dag, Ö. Highly Proton Conductive Phosphoric Acid-Nonionic Surfactant Lyotropic Liquid Crystalline Mesophases and Application in Graphene Optical Modulators. ACS Nano 2014, 8, 11007-11012.

(9) Olutaş, E. B.; Balcı, F. M.; Dag, Ö. Strong Acid-Nonionic Surfactant Lyotropic Liquid-Crystalline Mesophases as Media for the Synthesis of Carbon Quantum Dots and Highly Proton Conducting Mesostructured Silica Thin Films and Monoliths. Langmuir 2015, 31, 10265-10271.

(10) Çelik, Ö.; Dag, Ö. A New Lyotropic Liquid Crystalline System: Oligo(ethylene Oxide) Surfactants with $\left[\mathrm{M}\left(\mathrm{H}_{2} \mathrm{O}\right)_{\mathrm{n}} \mathrm{X}_{\mathrm{m}}\right.$ Transition Metal Complexes. Angew. Chem., Int. Ed. 2001, 40, 3800-3803.

(11) Albayrak, C.; Cihaner, A.; Dag, Ö. A New, Highly Conductive, Lithium Salt/Nonionic Surfactant, Lyotropic Liquid-Crystalline Mesophase and Its Application. Chem. - Eur. J. 2012, 18, 4190-4194.

(12) Albayrak, C.; Barım, G.; Dag, Ö. Effect of Hygroscopicity of the Metal Salt on the Formation and Air Stability of Lyotropic Liquid Crystalline Mesophases in Hydrated Salt-surfactant Systems. J. Colloid Interface Sci. 2014, 433, 26-33.

(13) Zakharova, L. Y.; Ibragimova, A. R.; Valeeva, F. G.; Kudryavtseva, L. A.; Konovalov, A. I.; Zakharov, A. V.; Selivanova, N. M.; Osipova, V. V.; Strelkov, M. V.; Galyametdinov, Y. G. Selforganization and Catalytic Activity of the Poly(ethylene Glycol)(10) Monododecyl ether/poly(ethyleneimine)/lanthanum Nitrate System. J. Phys. Chem. C 2007, 111, 13839-13845.

(14) Wang, L. M.; He, F.; Richert, R. Intramicellar Glass Transition and Liquid Dynamics in Soft Confinement. Phys. Rev. Lett. 2004, 92, 095701-4.

(15) Kato, T.; Yoshio, M.; Ichikawa, T.; Soberats, B.; Ohno, H.; Funahashi, M. Transport of Ions and Electrons in Nanostructured Liquid Crystals. Nat. Rev. Mater. 2017, 2, 17001.

(16) Yılmaz, E.; Olutaş, E. B.; Barım, G.; Bandara, J.; Dag, Ö. Lithium Salt-Nonionic Surfactant Lyotropic Liquid Crystalline Gel-Electrolytes with Redox Couple for Dye Sensitized Solar Cells. RSC Adv. 2016, 6, 97430-97437.

(17) Song, J. Y.; Wang, Y. Y.; Wan, C. C. Review of Gel-type Polymer Electrolytes for Lithium-ion Batteries. J. Power Sources 1999, 77, 183197.

(18) Tarascon, J. M.; Armand, M. Issues and Challenges Facing Rechargeable Lithium Batteries. Nature 2001, 414, 359-367.

(19) Rosol, Z. P.; German, N. J.; Gross, S. M. Solubility, Ionic Conductivity and Viscosity of Lithium Salts in Room Temperature Ionic Liquids. Green Chem. 2009, 11, 1453-1457.

(20) Croce, F.; Appetecchi, G. B.; Persi, L.; Scrosati, B. Nanocomposite Polymer Electrolytes for Lithium Batteries. Nature 1998, 394, 456-458.

(21) Polat, E. O.; Kocabas, C. Broadband Optical Modulators Based on Graphene Supercapacitors. Nano Lett. 2013, 13, 5851-5857.

(22) Barım, G.; Albayrak, C.; Yılmaz, E.; Dag, Ö. Highly Conducting Lyotropic Liquid Crystalline Mesophases of Pluronics (P65, P85, $\mathrm{P} 103$, and P123) and Hydrated Lithium Salts ( $\mathrm{LiCl}$ and $\mathrm{LiNO}_{3}$ ). Langmuir 2014, 30, 6938-6945. 
(23) Ferry, A.; Jacobsson, P.; Stevens, J. R. Studies of Ionic Interactions in Poly(propylene Glycol)4000 Complexed with Triflate Salts. J. Phys. Chem. 1996, 100, 12574-12582.

(24) Frech, R.; Chintapalli, S.; Bruce, P. G.; Vincent, C. A. Crystalline and Amorphous Phases in the Poly(ethylene Oxide) $-\mathrm{LiCF}_{3} \mathrm{SO}_{3}$ System. Macromolecules 1999, 32, 808-813.

(25) Cammarata, L.; Kazarian, S. G.; Salter, P. A.; Welton, T. Molecular States of Water in Room Temperature Ionic Liquids. Phys. Chem. Chem. Phys. 2001, 3, 5192-5200.

(26) Lightfoot, P.; Mehta, M. A.; Bruce, P. G. Crystal-Structure of the Polymer Electrolyte Poly(Ethylene Oxide) ${ }_{3} \mathrm{LiCF}_{3} \mathrm{SO}_{3}$. Science 1993, 262, 883-885.

(27) Volel, M.; Armand, M.; Gorecki, W. Influence of Sample History on the Morphology and Transport Properties of PEO-Lithium Salt Complexes. Macromolecules 2004, 37, 8373-8380.

(28) Zardalidis, G.; Ioannou, E.; Pispas, S.; Floudas, G. Relating Structure, Viscoelasticity, and Local Mobility to Conductivity in PEO/ LiTf Electrolytes. Macromolecules 2013, 46, 2705-2714.

(29) Kerr, R. L.; Miller, S. A.; Shoemaker, R. K.; Elliott, B. J.; Gin, D. L. New Type of Li Ion Conductor with 3D Interconnected Nanopores via Polymerization of a Liquid Organic Electrolyte-Filled Lyotropic Liquid-Crystal Assembly. J. Am. Chem. Soc. 2009, 131, 15972-15973.

(30) Lee, J. H.; Han, K. S.; Lee, J. S.; Lee, A. S.; Park, S. K.; Hong, S. Y.; Lee, J. C.; Mueller, K. T.; Hong, S. M.; Koo, C. M. Facilitated Ion Transport in Smectic Ordered Ionic Liquid Crystals. Adv. Mater. 2016, 28, 9301-9307.

(31) Nair, R. R.; Blake, P.; Grigorenko, A. N.; Novoselov, K. S.; Booth, T. J.; Stauber, T.; Peres, N. M. R.; Geim, A. K. Fine structure constant defines visual transparency of graphene. Science 2008, 320, $1308-1308$.

(32) Fang, H.; Bechtel, H. A.; Plis, E.; Martin, M. C.; Krishna, S.; Yablonovitch, E.; Javey, A. Quantum of Optical Absorption in TwoDimensional Semiconductors. Proc. Natl. Acad. Sci. U. S. A. 2013, 110, 11688-11691.

(33) Wang, F.; Zhang, Y. B.; Tian, C. S.; Girit, C.; Zettl, A.; Crommie, M.; Shen, Y. R. Gate-Variable Optical Transitions in Graphene. Science 2008, 320, 206-209. 